\title{
Novo gênero e nova espécie de Pseudachorutini do sudeste do Brasil (Collembola, Neanuridae, Pseudachorutinae)
}

\author{
Maria C. de Mendonça \& Liliane H. Fernandes
}

\begin{abstract}
Departamento de Entomologia, Museu Nacional, Universidade Federal do Rio de Janeiro. Quinta da Boa Vista, São Cristóvão, 20940-040 Rio de Janeiro, Rio de Janeiro, Brasil. E-mail: mcleide @acd.ufrj.br
\end{abstract}

\begin{abstract}
A new genus and a new species of Pseudachorutini from the southeastern Brazil (Collembola, Neanuridae, Pseudachorutinae). Tijucameria gen. nov. and T. mame sp. nov., are described and illustrated. The material was found on a ravine, in soil and retained litter among roots, at Parque Nacional da Tijuca, Rio de Janeiro, Brazil.
\end{abstract}

KEY WORDS. Atlantic rainforest, Brazil, Pseudachorutini.

RESUMO. Tijucameria gen. nov. e T. mame sp. nov., são descritos e ilustrados. O material foi encontrado em solo e "litter" retidos entre raízes, sobre barranco, no Parque Nacional da Tijuca, Rio de Janeiro, Brasil.

PALAVRAS CHAVES. Brasil, Mata Atlântica, Pseudachorutini.

Pseudachorutini inclui cerca de 300 espécies distribuídas em 27 gêneros, sendo 15 de ocorrência neotropical (BELlinger et al. 1996/ 2005). No Brasil, esta tribo está representada, atualmente, por 11 gêneros: Pseudachorutes Tullberg, 1871; Halachorutes Arlé, 1966; Pseudanurida Schott, 1901; Aethiopella Handschin, 1942; Arlesiella Delamare Deboutteville, 1951; Neotropiella Handschin, 1942; Brasilimeria Stach, 1942; Arlesia Handschin, 1942; Kenyura Salmon, 1954; Furculanurida Massoud, 1967 e Micranurida Borner, 1901. Destes, somente os gêneros Brasilimeria e Halachorutes foram descritos para o país.

Durante os trabalhos realizados no Parque Nacional da Tijuca, Rio de Janeiro, Rio de Janeiro, visando o estudo de biodiversidade de Collembola, encontraram-se inúmeros exemplares de Pseudachorutini, cuja singularidade dos caracteres permitiu diferenciar um novo gênero e uma nova espécie.

\section{Tijucameria gen. nov.}

Diagnose. Aspecto geral do corpo, alargado e achatado, paratergitos salientes e arredondados (tipo 3) sensu Massoud, 1967. Coloração acinzentada. Tegumento fortemente granuloso. Quetotaxia da cabeça e do corpo reduzida. Artículo antenal IV com vesícula apical, organito subapical, sensilas subcilíndricas. Órgão pós-antenal moruliforme, com vesículas apicalmente arredondadas. Seis olhos de cada lado da cabeça. Cone bucal fino e alongado. Maxila fina com duas lamelas. Mandíbulas denteadas. Tenáculo ausente. Furca reduzida com aspecto mamiliforme. Mucro presente. Segmento abdominal VI não visível dorsalmente.

Etimologia. Em alusão à localidade-tipo, Floresta da Tijuca.
Espécie-tipo: Tijucameria mame sp. nov.

Discussão. O novo gênero, Tijucameria, é muito próximo de Venezuelida Díaz \& Najt, 1994, no que se refere ao número de olhos, ao órgão pós-antenal moruliforme, à ausência de tenáculo e à presença de furca reduzida com aspecto mamiliforme. Comparando os referidos gêneros, diferenças marcantes foram observadas, principalmente no tocante à forma do corpo, às vesículas do órgão pós-antenal e à presença de um mucro. Em Venezuelida, segundo Díaz \& NajT (1994), as vesículas do órgão pós-antenal são apicalmente subdivididas, diferentemente de Tijucameria, que as apresenta apicalmente arredondadas; a furca embora tenha o mesmo aspecto nos gêneros aqui tratados, em Tijucameria é provida de um pequeno mucro em forma de bico. Além disto, o aspecto do corpo pseudachorutiforme em Venezuelida é bastante diferente do corpo ceratrimeriforme exibido por Tijucameria gen. nov. A tabela I exibe os principais caracteres diferenciais.

\section{Tijucameria mame sp. nov.}

Figs $1-9$

Comprimento do corpo variando de $1,5 \mathrm{~mm}$ a $2,25 \mathrm{~mm}$. Coloração acinzentada, uniforme. Tegumento granuloso. Revestimento de cerdas simples e curtas. Cerdas sensoriais lisas e longas cerca de sete vezes o comprimento de uma cerda simples (Fig. 1).

Antenas mais curtas que a diagonal cefálica. Artículo antenal IV com vesícula apical trilobada, cinco sensilas muito finas e subcilíndricas entre poucas cerdas finas e longas; um organito côncavo subapical alojado em fosseta; microsensila 


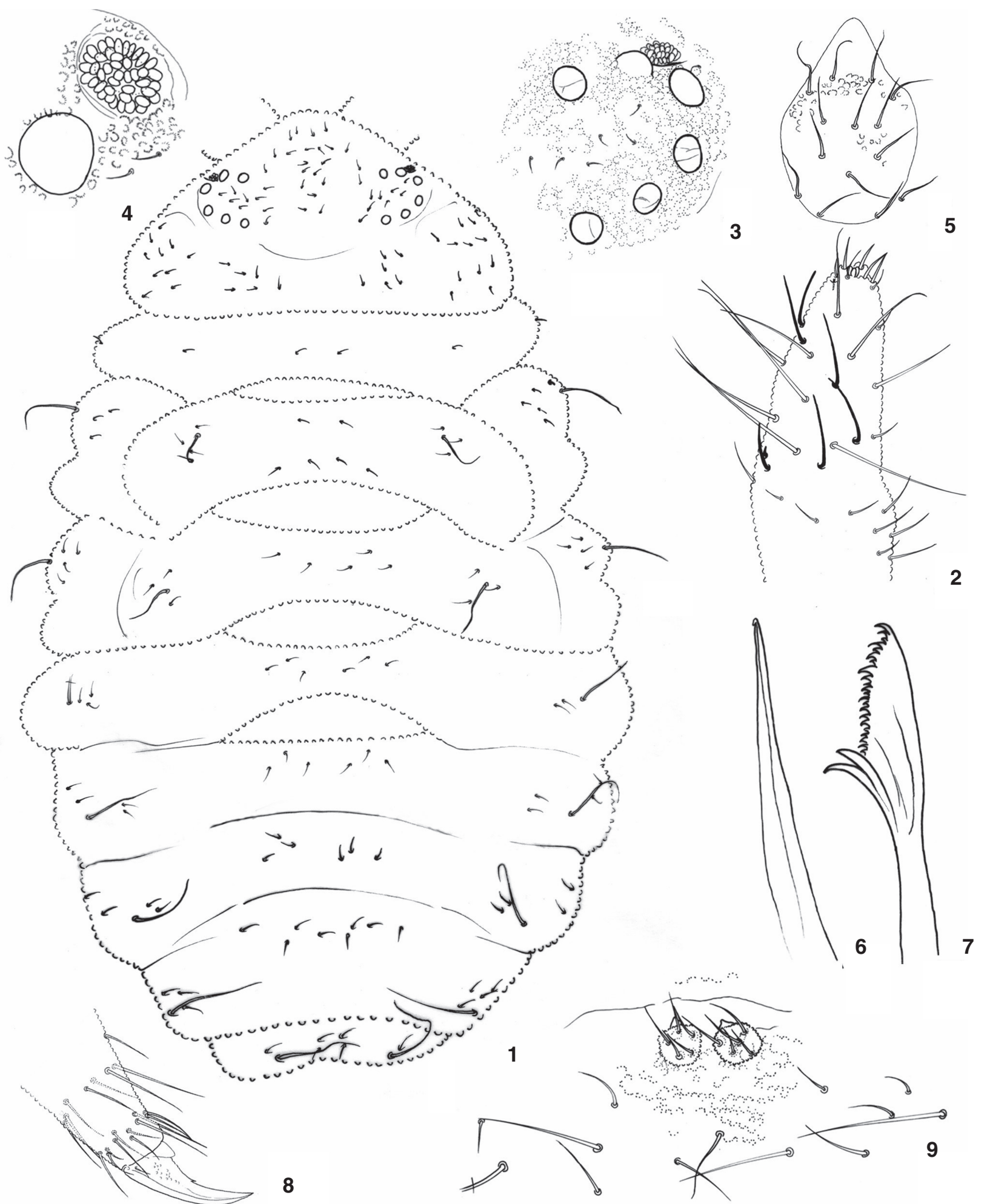

Figuras 1-9. Tijucameria mame sp. nov.: (1) quetotaxia dorsal; (2) face dorso-lateral da antena; (3) grupo ocular e órgão pós-antenal; (4) órgão pós-antenal e olhos; (5) labro e cerdas labrais; (6) maxila; (7) mandíbula; (8) tibiotarso e unha; (9) furca. 
Tabela I. Principais caracteres diferenciais entre os gêneros Tijucameria gen. nov. e Venezuelida.

\begin{tabular}{lll}
\hline \multicolumn{1}{c}{ Caracteres } & \multicolumn{1}{c}{ Tijucameria } & \multicolumn{1}{c}{ Venezuelida } \\
\hline Olhos & $6+6$ & $4+4$ \\
Vesículas do órgão pós-antenal & Apicalmente arredondadas & Apicalmente subdivididas em lóbulos \\
Fórmula labral & $4 / 3,3,2,2$ & $4 / 2,3,5,2$ \\
Furca & Mamiliforme com mucro & Mamiliforme sem mucro \\
Habitus & Ceratrimeriforme & Pseudachorutiforme \\
Coloração & Acinzentada & Branca \\
\hline
\end{tabular}

dorso-externa e "râpe" sensorial ventral ausentes. Órgão sensorial do artículo antenal III com duas sensilas pequenas e retas, protegidas por duas sensilas de guarda subcilíndricas, e uma microsensila ventral (Fig. 2). Artículo antenal I e II com 10 e 11 cerdas, respectivamente. Olhos em número de 6+6, medindo $17 \mu \mathrm{m}$; órgão pós-antenal, com diâmetro de $25 \mu \mathrm{m}$, composto de 36-43 vesículas com ápices arredondados (Figs 3 e 4). Cone bucal fino e alongado; fórmula labral 4/3,3,2,2 (Fig. 5); maxilas estiliformes com lamelas soldadas e extremidades em "crochet" (Fig. 6); mandíbulas robustas, medindo $40 \mu \mathrm{m}$, com 22-24 dentes sendo dois basais maiores que os demais (Fig. 7).

Tibiotarsos I, II e III com 19, 19, e 18 cerdas respectivamente, desprovidos de "ergot" capitado; unha forte, medindo $125 \mu \mathrm{m}$, com pequeno dente na crista interna, sem dentes laterais (Fig. 8). Tubo ventral com 3+3 cerdas. Tenáculo ausente. Furca reduzida, "dens" mamiliforme com cinco cerdas na face ventral; mucro reduzido, em forma de bico (Fig. 9). Segmento abdominal VI visível apenas ventralmente.

Os exemplares estudados mostraram-se adultos, embora não tenha sido possível determinar o sexo.

Holótipo. Brasil, Rio de Janeiro: Rio de Janeiro (Parque Nacional da Tijuca, Floresta da Tijuca), M.C. de Mendonça leg. Holótipo em lâmina No1220 CM/MNRJ, 19/IX/2002. Parátipos da mesma procedência: 4 exemplares em lâminas No1220 CM/MNRJ, 19/ IX/2002; 1 exemplar em lâmina Nº1383 CM/MNRJ, 28/VIII/2003;
1 exemplar em lâmina Nº1526 CM/MNRJ, 01/IX/2004; 1 exemplar em lâmina No1539 CM/MNRJ, 09/IX/2004. O material estudado está depositado na Coleção de Collembola, Departamento de Entomologia, Museu Nacional, Rio de Janeiro (CM/MNRJ).

Derivatio nominis. Dedicado à Maria da Conceição de Mendonça a quem chamávamos carinhosamente de mame (in memoriam).

\section{AGRADECIMENTOS}

Agradecemos ao Conselho Nacional de Desenvolvimento Científico e Tecnológico (CNPq) pela concessão de bolsa de estudo e à Fundação Carlos Chagas Filho de Amparo a Pesquisa do Estado do Rio de Janeiro (FAPERJ) pelo auxílio financeiro. Ao aluno de mestrado, Eduardo Assis Abrantes, pelo companheirismo demonstrado durante os trabalhos de campo e pelo apoio logístico computacional.

\section{REFERÊNCIAS BIBLIOGRÁFICAS}

Bellinger, P.F. ; K.A. Christiansen \& F. Janssens. 1996/2005. Checklist of the Collembola of the World. Available from World Wide Web at: http://www.collembola.org.

Diaz, A. \& J. NAjT. 1994. Collemboles (Insecta) des Andes vénézuéliennes. Bulletin du Muséum National d'Histoire Naturelle, Paris, 16 (2-4): 417-435.

Recebido em 10.I.2005; aceito em 16.VIII.2005. 